\title{
Memory for ideas: Synonym substitution
}

\author{
WILLIAM F. BREWER \\ University of Illinois at Urbana.Champaign, Champaign, Illinois 61820
}

\begin{abstract}
Subjects memorized sentences that contained a word with a synonym in English. There were large numbers of synonym substitution responses in recall (varying from $6.0 \%$ in Experiment $I$ to $27.7 \%$ in Experiment III). Synonym substitution responses tended to be unidirectional and occurred with roughly equal frequency in abstract and concrete sentences. The results were interpreted as opposed to surface structure theories of sentence memory, since explanation in terms of word associations and sequential dependencies proved unsuccessful. The results were also taken to be opposed to image theories of sentence memory, since image theories predict few if any synonym substitution responses for abstract sentences, while Experiment III found large numbers of synonym substitutions in both abstract and concrete sentences. The results support the position that memory for sentences is in terms of nonlinguistic, nonimage, abstract representations (ideas).
\end{abstract}

In 1894, Binet and Henri published a very significant paper on human memory in which they presented and interpreted the results of an extensive study of children's sentence recall. Binet and Henri pointed out that synonym substitutions rarely occurred in the recall of 11-word passages but were very common in the recall of 60- and 86-word passages. They interpreted this finding as showing that there are two kinds of memory-verbal memory and memory for ideas. In short passages the subject uses his verbal memory (memory for surface structure) to give back verbatim recall of the words presented. However, in longer passages the subject retains only the underlying ideas, so if the language has more than one lexical expression for this idea, the subject may select the "wrong" lexical form, giving a synonym substitution. Binet and Henri suggested that a process of "verbal assimilation" occurs when children recall prose material. In this process, children tend to replace the surface structure of the original material with lexical items and syntactic constructions more consistent with their own speech.

The title of Binet and Henri's paper, "La mémoire des phrases (Mémoire des idées)," clearly shows that they believed that linguistic material is stored in nonlinguistic form. However, it is difficult to be sure exactly what they meant by "idea," since this paper was written at a period when Binet was shifting from the position that mental processing is carried out exclusively with mental images to the position that mental processing is carried out predominately through imageless thoughts, as later proposed by the Wurzburg School (Reeves, 1967). For the purposes of this paper, the memory-for-ideas hypothesis will be interpreted to mean that information is stored in abstract, nonlinguistic, nonimage form.

The author thanks Richard Harris, Edward Lichtenstein, and Kathryn Bock for comments on an earlier draft of this paper. and Ken Schweller and Ellen Brewer for help with the data tabulation and analysis.

Requests for reprints should be sent to William F. Brewer, Department of Psychology, Psychology Building, University of Illinois, Champaign, Illinois 61820.
The purpose of the present paper is to extend Binet and Henri's empirical investigations of the synonym substitution phenomenon, to revive their theoretical position, and to draw the implications of this work for current theories of sentence memory.

While the synonym substitution phenomenon reported by Binet and Henri supports their memory-for-ideas approach, it is, without additional evidence, also compatible with a number of current theories of sentence memory. Surface structure theories (Johnson, 1968; Rosenberg, 1965) could, by a natural extension, argue that no process of abstraction and reconstruction is involved in recall and that the synonym substitutions are produced by high-strength word associations or by Markovian sequential dependencies. Syntactic theories of sentence memory (Mehler, 1963; Miller, 1962), however, have no well-motivated way to account for synonym substitutions. Image theories of sentence memory (Begg, 1971; Begg \& Paivio, 1969; Sasson, 1971) can deal with synonym substitutions in concrete sentences, since they assume that sentences are converted into mental images for storage and that these mental images are then encoded into words during recall. Thus, synonym substitutions will occur in recall when the subjects select some word other than the presented word for a particular mental image. Image theories of sentence memory assume that abstract sentences are not converted into mental images in memory, but are stored as "sequential verbal strings;" thus, there should be no synonym substitutions for abstract sentences. Memory-for-meaning theories (Fillenbaum, 1966; Sachs, 1967) are very similar to Binet and Henri's memory-for-ideas hypothesis and can deal with the synonym substitution phenomenon in much the same way as Binet and Henri did. The only major difference between the memory-for-ideas approach and the memory-for-meaning approach is that the idea hypothesis is somewhat more specific than the meaning hypothesis, since ideas are presumed to be nonlinguistic and nonimage in nature, whereas "meaning" has 
typically been completely unspecified. Thus, while Binet and Henri assumed that the phenomenon of synonym substitution itself was sufficient to support a memory-for-ideas position, a closer analysis shows that most current theories of sentence memory can handle their findings.

Experiment I was an attempt to develop experimental control over the synonym substitution phenomenon by constructing materials to produce synonym substitutions, rather than simply observing naturally occurring examples.

\section{EXPERIMENT I}

\section{Method}

Subjects. The subjects were 60 undergraduate students who participated in the experiment as part of a course requirement in introductory psychology.

Materials. The experimental materials consisted of 80 experimental sentences and 40 control sentences. Each experimental sentence contained one word for which there is a close synonym in English. The initial sample of synonyms used in the construction of the experimental sentences was obtained from a pool of approximately 4,000 synonym pairs chosen from Webster's Dictionary of Synonyms (1951). Each synonym pair was rated by three experienced raters on a 7-point scale, where 1 was "almost perfect similarity of meaning" and 7 was "very poor agreement in meaning." A pool of 385 close English synonyms was developed by retaining all synonym pairs with mean scores of 2 or less. These synonyms and a few additional close synonyms uncovered in pilot work were used to construct the experimental sentences. Each pair of experimental sentences was developed by writing a simple declarative frame into which either member of a synonym pair could be substituted to make an English sentence. Control sentences were written to be as similar to the experimental sentences as possible, except that the words around which these sentences were constructed did not have obvious synonyms. The 80 experimental sentences consisted of 40 sentence pairs, each resulting from the substitution of the two members of a synonym pair into a common sentence frame. An example of a sentence pair is The nightgown was too little, The nightgown was too small. The 40 synonym pairs were distributed across parts of speech: 17 verb pairs, 11 noun pairs, 11 adjective pairs, and 1 preposition pair.

Two 80-item master lists were developed. Fach master list contained one member of each of the 40 synonym sentence pairs and all 40 control sentences. Each master list was subdivided into four 20-jtem experimental lists such that each list contained 10 experimental sentences and 10 control sentences. The initial order of sentences in the experimental lists was random, but this order was consistent across the two sets of experimental lists derived from the two master lists, so that synonym pairmates and control itcms occurred in the same position on each experimental list. Five additional items were used to construct i practice list.

Design. Ten subjects received experimental lists derived from one master list and 10 subjects received experimental lists derived from the other master list. Thus, the 40 control sentences were heard by all 20 subjects, while an experimental sentence containing a particular synonym was heard by 10 subjects. There were two complete randomizations of order of items within master lists, with half the subjects receiving experimental lists derived from one randomization and half receiving experimental lists derived from the other.

Procedure. Each subject reccived a five-item practice list and four 20 -item experimental lists. The instructions stated that written recall would be required, but did not explicitly suggest cither a rote-memory or a gist-memory strategy. Thu experimenter read ach list of sentences aloud with normal
Table 1

Experiment I: Percentage of Sentences in Each Scoring Category for Experimental and Control Sentences

\begin{tabular}{|c|c|c|c|c|}
\hline Sentence Type & $\begin{array}{l}\text { Cor- } \\
\text { rect }\end{array}$ & $\begin{array}{r}\text { Scoring } \\
\text { Synonym }\end{array}$ & $\begin{array}{l}\text { ategory } \\
\text { Error }\end{array}$ & Omit \\
\hline $\begin{array}{l}\text { Experimental } \\
\text { Control }\end{array}$ & $\begin{array}{l}27.4 \\
34.0\end{array}$ & 6.0 & $\begin{array}{l}34.6 \\
36.4\end{array}$ & $\begin{array}{l}32.0 \\
29.6\end{array}$ \\
\hline
\end{tabular}

intonation at a rate of one sentence every $4 \mathrm{sec}$. After the last sentence in each list, the experimenter read four strings of sixand seven-digit numbers. Following each string of digits, the subjects wrote these numbers on the top page of their answer booklets. After they had written the last set of digits, subjects were asked to turn the page and write as many of the sentences from the most recent list as they could remember. Approximately $45 \mathrm{sec}$ elapsed between the reading of the last sentence and the beginning of the sentence-memory test. The answer booklets contained recall cues consisting of the initial noun phrase of each sentence in the same order as the acquisition lists. The subjects were given $4 \mathrm{~min}$ to recall the sentences.

Rating. Fifteen subjects rated the 40 synonym sentence pairs for naturalness. The subjects received booklets containing the sentence pairs and were asked to indicate which member of each pair they thought was "closest to the way you would say the sentence." Twenty-five additional subjects carried out a sentence-completion task. These subjects received the 40 experimental sentence frames with the synonyms omitted. They were asked to fill in the blank space in the sentence frame with the first word they could think of that would make a complete sentence.

\section{Results and Discussion}

The sentences written by the subjects in the recall task were classified into one of four mutually exclusive categories: (a) The category "correct" was used if the sentence was written exactly as presented. (b) The category "synonym substitution" was used for experimental sentences if the sentence met the criteria for correct except that the predicted synonym was written in place of the presented synonym. Thus, for the presented sentence The nightgown was too little, the response The nightgown was too small would be scored as a synonym substitution, whereas, for the presented sentence The nightgown was too small, the response The nightgown was too little would be scored as a synonym substitution. (c) The category "omit" was used when nothing was written after the recall cue. (d) The category "error" was used for all other responses.

Table 1 gives the percent of responses in each of the scoring categories for experimental sentences and for control sentences. As in Binet and Henri's study, there were a number of synonym substitutions.

However, the overall results $(6 \%$ synonym substitutions) were not as strong as might be expected from the hypothesis that sentences are stored as nonlinguistic ideas and then transformed back into linguistic form during recall. A closer analysis of the data suggested several difficulties with Experiment I that may have reduced the strengtli of the synonym substitution phenomenon. There were no synonym substitutions at all in 50 of the 80 experimental sentences. Yet a small number of sentences showed a very robust effect-one 
showed 5 shifts out of 10 possible, another 4 out of 10 , and three gave 3 out of 10 . This suggested the need for better selection of experimental items. In the design of Experiment I, it had been assumed that synonym substitution would be a bidirectional phenomenon, with one form shifting to the other about as frequently as that second form shifted to the first. However, the results of the experiment showed that, out of the 25 synonym sentence pairs with at least one synonym shift, only five showed bidirectional shifts, and, of these, several were asymmetric (for example, five shifts of The hippo frightened the children to The hippo scared the children but only one shift in the opposite direction).

A number of variables obtained from the rating data were examined in an attempt to account for the unidirectional nature of the shifts. A naturalness score for each synonym sentence was obtained by taking the number of subjects (out of 15 ) who chose that sentence as the more natural member of the pair. A sentence-completion score for each synonym sentence was obtained by calculating the number of times (out of 25 opportunities) that the sentence was produced by subjects filling in the missing synonym word in the sentence frame. In addition, the frequency of occurrence of each of the synonym words was obtained (Kučera \& Francis, 1967). The frequency score for each sentence was the frequency of its synonym as a percentage of the total frequency of both synonyms. The means for naturalness, sentence completion, and frequency for the shifting and nonshifting members of the 20 unidirectionally shifting pairs are given in Table 2 . The results show that the synonym substitutions are from the less natural forms to the more natural forms, $t(19)=5.42 . p<.01$ : and from the less frequently completed forms to the more frequently completed forms, $t(19)=5.52, p<.01$. There was no significant tendency for the member of a pair with the less frequent synonym to shift to the form with the more frequent synonym. These results suggest that the process which Binet and Henri called "verbal assimilation" occurs for adults as well as children. When the subjects attempted to reconstruct the surface form of the original sentences, certain characteristics of their speech output systems operated to produce systematic biases in the particular surface forms that were given in recall. Since synonym substitution tends to be a unidirectional phenomenon, the use of counterbalanced lists containing both forms reduces the observed strength of the effect because for many of the sentences (the nonshifting members of unidirectionally shifting sentence pairs) there will be no

Table 2

Experiment I: Mean Naturalness Scores, Sentence Completion Scores, and Frequency Scores for the Shifting and Nonshifting Member of Each Synonym Sentence Pair

\begin{tabular}{cccc}
\hline & \multicolumn{3}{c}{ Scores } \\
Sentence Type & Naturalness & Frequency & Completion \\
\hline Nonshifting & 11.7 & 48.6 & 8.4 \\
Shifting & 3.4 & 51.5 & 1.9 \\
\hline
\end{tabular}

synonym shifts. Thus, for Experiment II additional sentences were written, and only the unidirectionally shifting members of the synonym sentence pairs were presented to the subjects.

\section{EXPERIMENT II}

\section{Method}

Subjects. The subjects were 70 undergraduate students who participated in the experiment as part of a course requirement in introductory psychology.

Materials. The experimental materials consisted of 30 experimental sentences and 30 control sentences. Each experimental sentence contained one word for which there is a close synonym in English, as in Experiment I. However, only one member of a synonym sentence pair was used in this experiment. The sentence chosen from each pair was the sentence that had shown the strongest tendency to give recall shifts in earlier experiments. Three 20 -item experimental lists were developed by randomly selecting items from the pool of 30 experimental and 30 control items such that each experimental list contained 10 experimental sentences and 10 control sentences. An additional 20 items were used to construct a practice list.

Design and procedure. Each of 30 subjects received 80 sentences ( 20 practice sentences, 30 experimental sentences, 30 control sentences). There were three different randomizations of order of items with 10 subjects in each. Other than these changes, the instructions and procedure were identical with those in Experiment 1 .

Rating. Fifteen subjects rated the 30 synonym pairs (the 30 experimental sentences plus the 30 sentences to which they were expected to shift) for naturalness, as in Experiment $I$. Twenty-five additional subjects carried out a sentence-completion task with the 30 experimental sentence frames, as in Experiment I.

\section{Results and Discussion}

The sentences written by the subjects were classified into one of five mutually exclusive categories. The categories correct, synonym substitution, error, and omit were as described in Experiment I. The category "other synonym substitution" was used for sentences that met the criteria for the category "synonym substitution," but where the substituted word or phrase was not the particular one that had been predicted. The category other synonym substitution was applied to both experimental and control sentences. This category was necessary, since English contains more than two lexical items appropriate to some of the ideas underlying the experimental sentences. Thus, the sentence The Indian was hiding below (under) the bridge was sometimes recalled as The Indian was hiding beneath the bridge, while the sentence The surfer almost lost his swimming suit (swimming trunks) was sometimes recalled as The surfer almost lost his bathing suit.

Table 3 gives the percent of responses in each of the scoring categories for experimental sentences and for control sentences. Sentences that contain a word that has a potential synonym in English frequently shift to a form containing that synonym in recall $(20.8 \%$ synonym substitution) and are consequently very difficult to 
Table 3

Experiment II: Percentage of Sentences in Each Scoring Category for Experimental and Control Sentences

\begin{tabular}{lccccc}
\hline $\begin{array}{c}\text { Sentence } \\
\text { Type }\end{array}$ & $\begin{array}{c}\text { Cor- } \\
\text { rect }\end{array}$ & $\begin{array}{c}\text { Syno- } \\
\text { nym }\end{array}$ & $\begin{array}{c}\text { Other } \\
\text { Synonym }\end{array}$ & Error & Omit \\
\hline Experimental & 16.4 & 20.8 & 5.3 & 30.4 & 27.0 \\
Control & 48.2 & & .8 & 27.6 & 23.4 \\
\hline
\end{tabular}

recall verbatim (only $16.4 \%$ correct).

Experiment II shows that synonym substitution is a very robust phenomenon once experimental control has been obtained. Surface structure theories of sentence memory (Johnson, 1968; Rosenberg, 1965) would seem to have at least two possible explanations for these results-word associations and sequential dependencies. The word-association approach would assume that no abstract transformation of the input is occurring and that the synonym substitutions are simply word associations occurring to the original item, either during presentation or during recall. There are two major difficulties with this hypothesis. First, the word-association norms frequently predict the wrong synonym substitution. For example, in the sentence The St. Bernard was too large for the car, the word large frequently shifted to big in recall, yet the highest associate for large is small, and small was never given in recall. Thus, the recall shifts preserve the underlying ideas or meaning, something that cannot be explained at the level of surface structure. The second difficulty is that the word-association hypothesis does not explain why high associates are not given for the nonsynonym words in the sentences. For example, the sentence The gold statue was in the center (middle) of the square contains the nonsynonym word square. The word-association norms show that round is the highest associate of square, yet the sentence The gold statue was in the center of the round never occurred in recall.

The sequential dependency approach to the synonym substitution phenomenon would assume that these responses are produced when the subject retains all of the surface structure of the presented sentence except for the synonym item and then simply fills in the most probable word. There are a number of fundamental difficulties with this explanation. First, like the word-association approach, it does not explain where the probabilities come from and does not give a wellmotivated reason why only the synonym words are so frequently changed. In addition, the sentence-completion data for those sentence frames with little semantic constraint go against this hypothesis. Thus, sentences such as The girl selected (chose) a platinum ring and The nightgown was too little (small) showed a large number of synonym shifts (to the word in parenthesis). Yet, when given as sentence frames in the sentence-completion task The girl a platinum ring and The nightgown was too ), the synonym substitution word was rarely given as a response. It appears that subjects who have heard the original sentence make the synonym shifts because they have retained the underlying ideas or meaning, whereas subjects who attempt to complete a fairly unconstrained sentence do not know what the original meaning was, so they rarely respond with the synonym word.

While the results of Experiment II are not compatible with surface structure theories of sentence memory, they are compatible with theories that postulate that sentences are stored in memory in terms of images, meanings, or ideas. Each of these theories suggests that the linguistic material is transformed into some abstract form in memory, and thus each predicts that sentences containing a word with a potential synonym will be difficult to remember verbatim, since many of the responses will express the underlying abstract information with the synonym rather than with the presented word.

While the results of Experiment II show that the synonym sentences are hard to remember verbatim, these sentences are only slightly inferior to the control sentences in terms of the recall of the underlying information, as might be expected from the image, meaning, or idea approaches. In the experimental sentences the subjects retained the underlying information in $42.6 \%$ of the responses (correct plus synonym substitution plus other synonym substitution), while in the control sentences the subjects retained the underlying information in $49.0 \%$ of the responses (correct plus other synonym substitution).

In comparing the proportion of corrects to synonym substitutions, the unidirectional nature of the synonym substitution phenonemon must be taken into account. If subjects retained only the idea underlying a particular presented word and no surface information about the word itself, and if the two synonyms chosen for the experiment were the only available words in English for that idea, and it both members of the synonym parr were equally available, then in recall the number of corrects and the number of synonym shifts should be roughly equal. However, in practice, probably none of these assumptions is correct, so that the obtained values of correct responses (16.4\%) and synonym substitutions $(20.8 \%)$ are the result of the interaction of a number of factors. It seems unlikely that subjects retain no surface information about sentences heard 1 to $6 \mathrm{~min}$ previously. Thus, there should be a bias toward recalling the original presented word of a synonym pair. However, presenting only the unidirectionally shifting member of a pair should produce a bias against recalling the original presented word. The fact that there were more synonym substitutions than correct responses suggests that in the present study the unidirectional bias was greater than the bias produced by retained surface information. 
In discussing the implications of this experiment for theories of sentence memory, the image approach has been included with the meaning and idea approaches as a viable theoretical framework. This is perhaps unfair to the meaning and idea approaches, since there are wellknown difficulties with the image theory which make this approach untenable. For example, it is difficult to see how an image approach can handle the syntactic relations of a sentence; it is also difficult to see how the subject decodes the mental image into the appropriate words: If the subject has stored an image for the word St. Bernard, how does he know that in recall he should give St. Benard rather than other words appropriate to the image, such as $d o g$ or animal? Further elaboration of these logical difficulties can be found in Brewer (in press) and Price (1969).

However, if one ignores these logical problems, then the results of Experiment II can be accounted for within an image framework, since most of the sentences used in Experiment II were concrete. Well-developed image theories of sentence memory (Begg \& Paivio, 1969; Paivio, 1971) make a qualitative distinction between memory for concrete sentences and memory for abstract sentences: Concrete sentences are retained as mental images, whereas abstract sentences are retained in terms of their surface structure. This distinction allows a very clear test of image theories of sentence memory: Since abstract sentences are not transformed into some abstract representation for storage, there should be few, if any, synonym substitutions in the recall of abstraci sentences. The idea and meaning approaches to sentence recall assume that the linguistic input of both concrete and abstract sentences is transformed into an abstract representation in memory and so predict that there should be little or no difference between abstract and concrete sentences in the number of synonym substitutions. Experiment III was designed to test the clear differential prediction made by the two classes of theories.

\section{EXPERIMENT III}

\section{Method}

Subjects. The subjects were 42 undergraduate students who participated in the experiment as part of a course requirement in introductory psychology.

Materials. The materials consisted of 20 concrete experimental sentences, 20 abstract experimental sentences, and 8 filler sentences. Each experimental sentence contained one word for which there is a close synonym in English, as in the previous experiments. The concrete and abstract sentences were obtained by developing additional abstract items and then scaling a heterogeneous sample of 48 sentences for imageability. Twelve subjects rated each sentence from 1 to 7 on the "ease or difficulty with which it arouses a mental image" ( 1 was the extreme low imagery end of the scale, 7 the extreme high imagery end). The 20 sentences with mean rating scores less than 3.7 were considered to be abstract sentences. The 20 sentences with mean rating scores greater than 4.2 were considered to be
Table 4

Experiment III: Percentage of Sentences in Each Scoring Category for Abstract and Concrete Sentences

\begin{tabular}{llcccc}
\hline $\begin{array}{c}\text { Sentence } \\
\text { Type }\end{array}$ & $\begin{array}{c}\text { Cor- } \\
\text { rect }\end{array}$ & $\begin{array}{c}\text { Syno- } \\
\text { nym }\end{array}$ & $\begin{array}{c}\text { Other } \\
\text { Synonym }\end{array}$ & Error & Omit \\
\hline Abstract & 26.7 & 23.0 & 5.8 & 29.3 & 15.2 \\
Concrete & 30.3 & 27.7 & 2.8 & 27.2 & 12.0 \\
\hline
\end{tabular}

concrete sentences. The mean rating score for all 20 abstract sentences was 2.46 , while the mean rating score for the 20 concrete sentences was 5.42 .

Four 12 -item experimentil lists were developed by randomly selecting items from the total pool of 20 concrete. 20 abstract. and 8 filler sentences such that each experimental list contained 5 concrete experimental sentences, 5 abstract experimental sentences, and 2 filler sentences. Twelve additional items were used to construct a practice list.

Design and procedure. All 30 subjects received a 12-item practice list and four 12-item experimental lists. Sentences were read at a rate of approximately one every $6 \mathrm{sec}$, and the subjects were given 3 min to recall the sentences. Other than these changes, the instructions and procedure were identical with those in Experiment II.

\section{Results and Discussion}

The sentences written by the subjects were classified as correct, synonym substitution, other synonym substitution, error, and omit, using the criteria described in Experiment II.

Table 4 gives the percent of responses in each of the scoring categories for abstract sentences and for concrete sentences. Large numbers of synonym substitution responses were made in the recall of both abstract $(23.0 \%)$ and concrete $(27.7 \%)$ sentences. In fact, if one calculates the number of shifts (synonym substitution plus other synonym substitution) as a percent of the responses in which the meaning was retained (correct plus synonym substitution plus other synonym substitution), there were slightly more shifts for abstract sentences (51.9\%) than for concrete sentences (50.2\%). Thus, the results of Experiment III provide support for idea and meaning theories of sentence memory and strong evidence against image theories of sentence memory.

There have been a large number of recent experiments supporting an image theory of sentence memory (Begg, 1971; Begg \& Paivio, 1969; Paivio, 1971; Sasson, 1971; Sasson \& Fraisse, 1972; Yuille \& Paivio, 1969). However, the results of the present experiment suggest that these experiments should be reinterpreted. Brewer (in press) has argued for an interactive view of sentence memory: Sentences (both abstract and concrete) are remembered in terms of their underlying ideas, but once a concrete sentence has been understood, the information can be used to construct a mental image which provides an additional mode of storage for some of the information contained in the sentence. This 
interactive approach thus provides for an abstract representation of the meaning of both concrete and abstract sentences and suggests that retention of concrete sentences should benefit from the additional information in the image store. A theoretical account of this type is capable of dealing with the results of the present experiment and with the finding that abstract linguistic material is generally harder to remember than concrete linguistic material.

However, the results of the Begg and Paivio (1969) experiment remain incompatible with the interactive approach to sentence memory. Begg and Paivio found that for concrete sentences subjects were better able to detect changes in meaning than in words, but for abstract sentences the reverse held. They interpret this result as lending support to the position that concrete sentences are remembered in terms of mental images, while abstract sentences are remembered in terms of their surface structures (i.e., as strings of words). This finding is incompatible with the interactive view outlined above, since the interactive view assumes that abstract words are represented in terms of their underlying ideas, so subjects should have been able to detect changes in meaning for abstract sentences, just as they could for concrete sentences. However, it appears that Begg and Paivio's results may be due to a systematic bias in the construction of the materials. Johnson, Bransford, Nyberg, and Cleary (1972) have shown that the abstract sentences used in the Begg and Paivio study are more difficult to comprehend than the concrete sentences. A comparison of the abstract sentences used in the Begg and Paivio study and those used in the present experiment certainly appears to support the analysis of Johnson et al. Compare Begg and Paivio's sample abstract sentence, The innocent occasion promoted a useless illusion, with typical abstract sentences from the present study, Russian is difficult (hard) to pronounce or The voters received (got) what they deserved. Thus, it seems likely that Begg and Paivio's findings are due to the fact that the subjects had difficulty in finding an appropriate set of underlying ideas or meanings for the abstract sentences.

Since the studies showing that concrete material is easier to remember than abstract material (Yuille \& Paivio, 1969) may also have confounded comprehension with abstractness-concreteness, it is difficult to know how much advantage imagery per se contributes. The results of the present study suggest that the effect is not very large. In writing the abstract sentences, an at tempt was made to make them as understandable as the concrete sentences. Table 4 shows that the underlying meanings or ideas (correct plus synonym substitution plus other synonym substitution) were recalled correctly in $55.5 \%$ of the abstract sentences and in $60.8 \%$ of the concrete sentences. So, to the extent that the abstract sentences and concrete sentences were equivalent in comprehension, the results suggest little advantage in recall for sentences that are imageable. Overall, the present study shows that synonym substitutions occur frequently in abstract sentences and that concrete sentences may be somewhat easier to recall than abstract sentences. These findings are compatible with a two-process approach which assumes that both concrete and abstract sentences are remembered in terms of their underlying ideas and that the imageability of concrete sentences may facilitate their recall.

\section{GENERAL DISCUSSION}

The basic empirical findings of this series of experiments are (a) it is possible to gain experimental control over the synonym substitution phenomenon; (b) synonym shifting tends to be unidirectional; (c) synonym substitutions occur with roughly equal frequency in both abstract and concrete sentences. The studies of Fillenbaum (1966) and Sachs (1967) showed that recognition memory for the meaning of sentences is superior to the recognition memory for surface structure; the present study shows that sentence recall conserves meaning better than surface structure. In Experiments II and III the phenonemon is particularly striking, since the subjects are more likely to write down a list they never heard (the shifted forms) than they are to write down the presented sentences.

An issue not resolved by this paper is what causes some synonym pairs to shift in recall, while others do not. The results of pilot work for the present study suggest that a wide variety of factors may be operating. For example, the word street never shifted to road, or vice versa. It may be that the lack of recall shifts is an indication that there really is a difference in meaning-streets are in cities, while roads are in the country. The word intoxicated never shifted to drumk. In this pair the meaning may be very similar, but the surface characteristics of the words are so different that they block the shift. Clearly, the synonym substitution technique can be used to study a number of attributes of lexical forms.

The results of the present experiments provide a basis for choosing among current theories of sentence memory. Surface structure theories (Johnson, 1968; Rosenberg, 1965) do not provide an abstract level of analysis for the representation of words in memory and must attempt to account for synonym shifts in terms of word associations or sequential dependencies. Attempts to account for the present data with these mechanisms were not successful. Syntactic theories of sentence memory (Mehler, 1963; Miller, 1962) provide no discussion of the representation of a particular lexical item and thus provide no well-motivated explanation of the results of these experiments. Image theories of sentence memory (Begg \& Paivio, 1969) assume that 
concrete sentences are stored as mental images, while abstract sentences are stored as strings of words. This position makes the strong prediction that synonym substitutions should occur for concrete sentences, but not for abstract sentences. The results of Experiment III show that there is little difference in abstract and concrete sentences in the occurrence of synonym substitution and thus stand as strong evidence against an image theory of sentence memory. Theories of memory for meaning (Fillenbaum, 1966; Sachs, 1967) are, of course, compatibie with the results of these experiments. However, $\mathrm{B}_{1}$ ewer (in press) has argued for the reintroduction of "idea" as a technical term in memory research, in order to specify that the postulated theoretical entities are nonlinguistic and nonimage in nature (see also Bransford \& Franks, 1971). It is clear that some way must be found to give structure to these underlying theoretical entities. However, the problem of providing a precise specification for meanings has thus far proved very resistant to solution, even after attack by generations of philosophers, linguists, and psychologists, and there is no attractive solution on the horizon.

Thus, in summary, the results of these experiments are best dealt with by a memory-for-ideas approach to sentence memory in which it is assumed that sentences are remembered in terms of their underlying ideas, but with parallel storage of some image information and some retained surface information. If enough surface information is retained or if there is only one obvious linguistic realization of a given set of ideas, then recall will tend to be verbatim correct, without any indication that a complex process of abstraction and reconstruction has occurred. However, if the sentence to be recalled contains the nonpreferred member of a synonym pair, then the underlying ideas will frequently be lexicalized with the "wrong" word, the resulting synonym-substitution response providing evidence for the intervening process of abstraction and reconstruction.

\section{REFERENCES}

Begg, I. Recognition memory for sentence meaning and wording. Journal of Verbal Learning and Verbal Behavior, 1971, 10, 176-181.

Begg, I., \& Paivio, A. Concreteness and imagery in sentence meaning. Journal of Verbal Learning and Verbal Behavior, $1969,8,821-827$.

Binet, A., \& Henri, V. La mémoire des phrases (Mémoire des idées). L'Année Psychologique, 1894, 1, 24-59.

Bransford, J. D., \& Franks, J. J. The abstraction of linguistic ideas. Cognitive Psvchologv. 1971.2. 331-35n

Brewer, W. F. The problem of meaning and the interrelations of the higher mental processes. In W. B. Weimer and D. S. Palermo (Eds.), Cognition and the symbolic processes. Potomac, Md: Erlbaum, in press.

Fillenbaum, s. Memory ror gist: Some relevant variables. Language and Speech, 1966, 9, 217-227.

Johnson, M. K., Bransford, J. D., Nyberg, S. E., \& Cleary, J. J. Comprehension factors in interpreting memory for abstract and concrete sentences. Journal of Verbal Learning and Verbal Behavior, $1972,11,451-454$.

Johnson, N. F. Sequen tial verbal behavior. In T. R. Dixon and D. L. Horton (Eds.), Verbal behavior and general behavior theory, Englewood Cliffs, N.J: Prentice-Hall, 1968. Pp. 421-450.

Kucera, H., \& Francis, W. N. Computational analysis of present-day American English. Providence, R.I: Brown University Press, 1967.

Mehler, J. Some effects of grammatical transformations on the recall of English sentences. Journal of Verbal Learning and Verbal Behavior, 1963. 2. 346-351.

Miller, G. A. Some psy chological studies of grammar. American Psychologist, 1962, 17, 748.762.

Paivio, A. Imagery and verbal processes. New York: Holt Rinehart and Winston, 1971.

Price, H. H. Thinking and experience, (2nd ed.) London: Hutchinson. 1969 .

Reeves, J. W. Thinking about thinking. New York: Delta, 1967.

Rosenberg, $S$. The influence of grammatical and associative habits on verbal learning. In S. Rosenberg (Ed.), Directions in psycholinguistics. New York: Macmillan, 1965. Pp. 121-145.

Sachs, J. S. Recognition memory for syntactic and semantic aspects of connected discourse. Perception \& Psychophysics, $1967,2,437-442$.

Sasson, R. Y. Interferring images at sentence retrieval. Journal of Experimental Psychology, 1971, 89, 56-62.

Sasson, R. Y. Interfering images at sentence retrieval. Journal of Experimental Psy chology, 1971, 89, 56-62. 1972. Y4. 14y-1bo.

Webster's dictionary of synonyms. Springfield, Mass: Merriam, 1951.

Yuille. J. C.. \& Paivio, A. Abstractness and recall of connected discourse. Journal of Experimental Psychology, 1969, 82, 467-471.

(Received for publication July 26, 1974; accepted September 9, 1974.) 